\title{
Complication Rates Relating to the Degree of Displacement of Femoral Neck Fractures: a Clinical Study of 878 Internally Fixed Intracapsular Fractures
}

\author{
Christopher R. Lawrence ${ }^{1}$ and Martyn J. Parker ${ }^{2}$ \\ ${ }^{1}$ Department of Orthopaedics, Broomfield Hospital, Chelmsford, Essex CM1 7ET, UK \\ ${ }^{2}$ Department of Orthopaedics, Peterborough City Hospital, Peterborough PE3 9GZ, UK \\ Address correspondence to Martyn J.Parker,martyn.parker@pbh-tr.nhs.uk
}

Received 8 December 2010; Revised 5 May 2011; Accepted 11 May 2011

\begin{abstract}
We undertook this large prospective study of intracapsular fractures treated with internal fixation to evaluate whether the degree of initial fracture displacement correlated to fracture healing complications including nonunion and avascular necrosis. We classified intracapsular fractures based on anteroposterior and lateral radiographs, as undisplaced, partially displaced and fully displaced. In total 878 patients with a non-pathological intracapsular hip fracture included in this study were treated by fixation of the fracture with three cancellous screws. Overall complications of fracture healing were $23.2 \%$ for undisplaced, $31.1 \%$ for partially displaced and $45.6 \%$ for fully displaced fractures showing correlation between fracture displacement and complication rate. The difference between the three groups for the complications of non-union and total fracture healing complications was statistically significant with an increasing complication rate as the fracture became more displaced. Intracapsular hip fractures show a progressive increase in the occurrence of fracture healing complication with increasing degree of initial fracture displacement.
\end{abstract}

\section{Introduction}

Fractures of the femoral neck (intracapsular hip fractures) constitute a common and expensive injury for health services to manage. Due to the tenuous blood supply to the femoral head and the difficulty in achieving stability after internal fixation, these fractures are associated with the complications of fracture displacement (or re-displacement after reduction and fixation), non-union and avascular necrosis [11].

Previous studies have suggested that the greater the degree of initial fracture displacement the greater the risk is for these fracture healing complications to occur $[1,9]$. This high risk of fracture healing complications has led to a significant proportion of these fractures being treated with a replacement arthroplasty. For those fractures with no, or minimal, displacement internal fixation is still preferred as this operation is less invasive and allows the patients to retain their own femoral head. The problem remains in how to define the degree of displacement that leads to an unacceptably high risk of fracture healing complications.

Current classification methods all concentrate on the appearance of the fracture on the anteroposterior (AP) radiograph $[9,15,19]$, with little consideration of the fracture on the lateral radiograph. A previous description of intracapsular fractures using radiographs and pathological specimens by Per Linton in 1949 [12] described the progressive degrees of displacement that may occur with intracapsular fractures (see Figure 1). This he termed the intermediary fracture pattern. The diagram shows how there may be minimal displacement on the AP radiograph with a range of angulation on the lateral radiograph and hence fragment separation.

This study uses a large series of patients with an intracapsular hip fracture all treated by internal fixation, and concentrates on the appearance of the fracture at presentation on both the anteroposterior and lateral radiographs. This, taken in conjunction with the principle that there is a continuous range of degree of displacement of the fracture, was used to subdivide a series of patients into groups of varying fracture displacement and to determine the outcome for these patients with regard to the occurrence of subsequent fracture healing complications following internal fixation. The complication rate in comparison with the measured angulation of the fracture was also assessed.

\section{Patients and methods}

Between January 1989 and August 2008, 3284 patients with an intracapsular hip fracture were treated at Peterborough District Hospital and were entered prospectively into a database of all hip fractures. In total 73 patients with a pathological fracture from tumor were excluded 

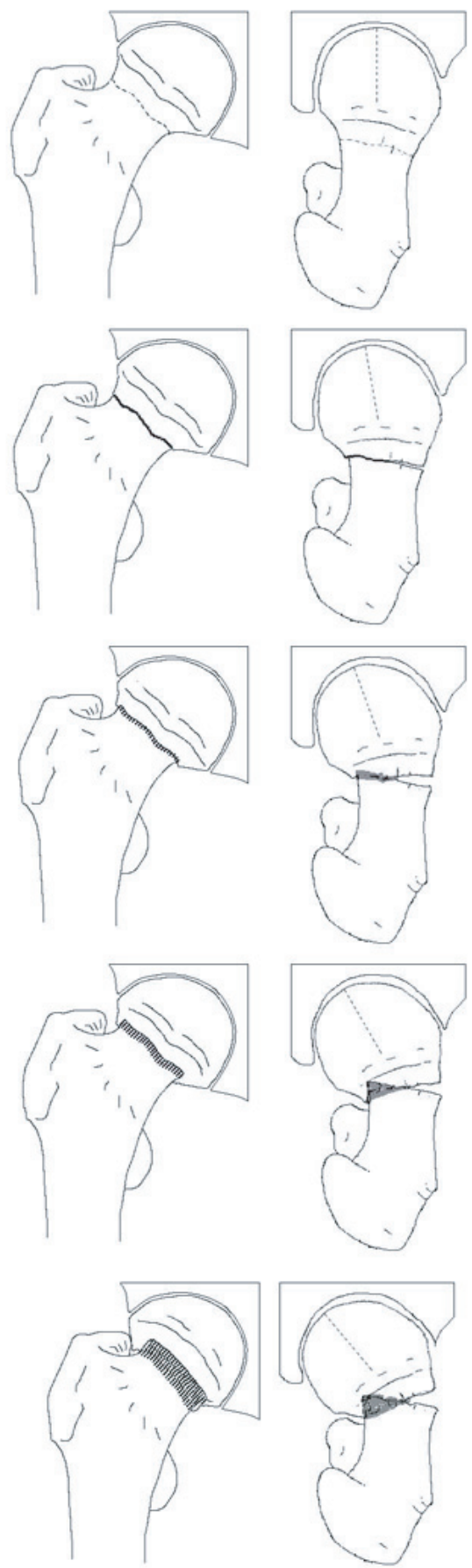

Figure 1: Degrees of displacement of intracapsular fractures showing the increasing angulation that occurs on the lateral radiograph (redrawn from [12]).

from further study. From the admission AP and lateral radiographs, fractures were divided into three categories: undisplaced, partially displaced and fully displaced. Undisplaced fractures were defined as those fractures that showed no displacement at the cortical fracture line on either the AP or lateral radiograph. Included in this

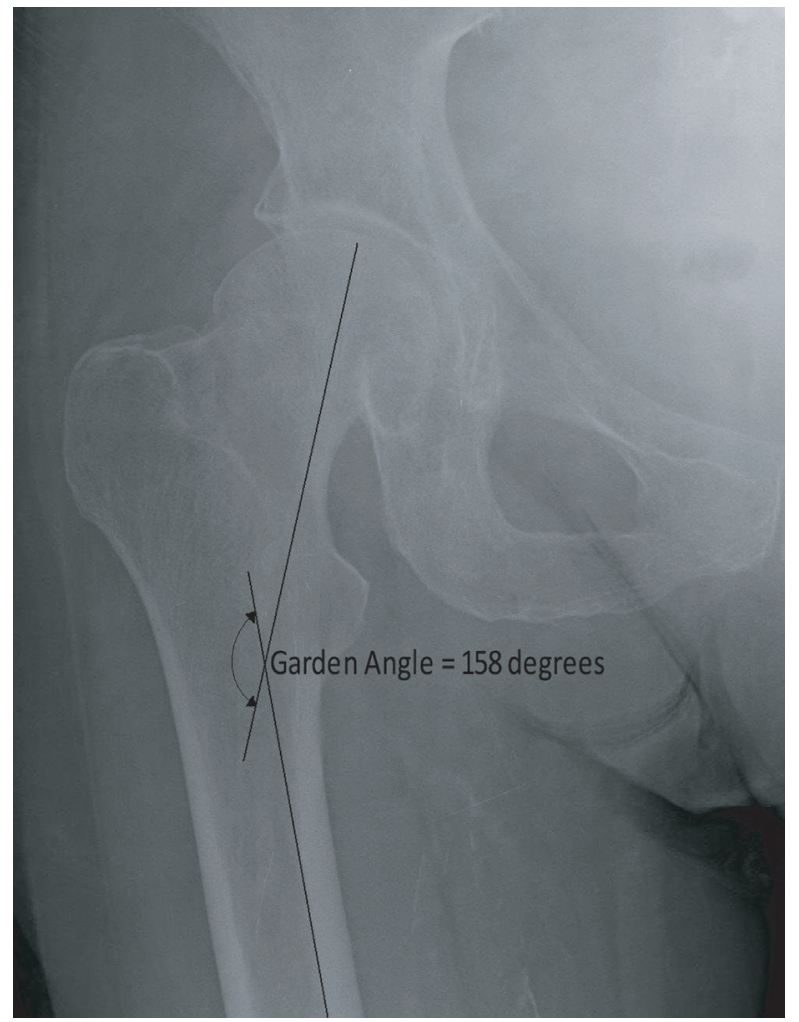

Figure 2: Anteroposterior radiographs of an intracapsular hip fracture with no definite displacement on the AP view (normal Garden alignment index angle $=160^{\circ}$ ).

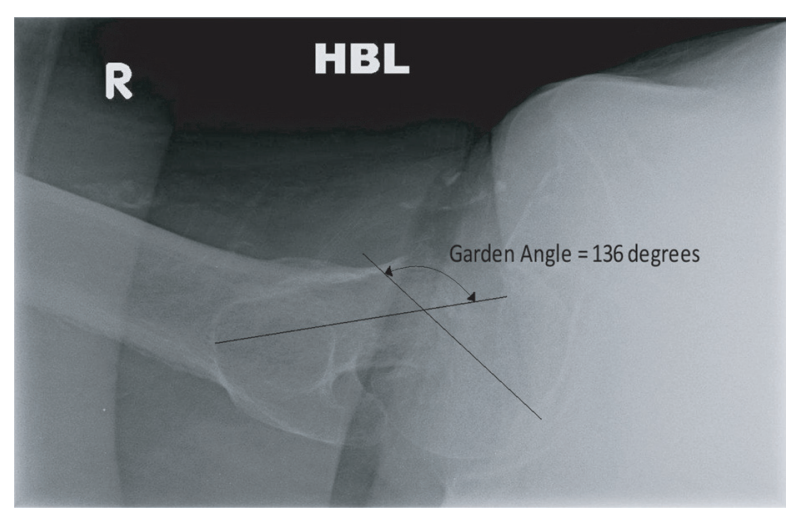

Figure 3: Horizontal-beam lateral radiograph of the same fracture shown in Figure 2 displaying a significant angulation at the fracture site (normal Garden alignment index angle $=180^{\circ}$ ).

category were those fractures with apparent impaction on the AP view. Partially displaced fractures were those that were undisplaced on only one of either the AP or lateral view (Figures 2 and 3). Fully displaced fractures showed displacement of the fracture on both the AP and lateral views. This categorization was performed by the senior 
author. As well as assessment based on displacement, a measurement of the trabecular angle on the lateral radiograph was also recorded, the normal angle being 180 degrees.

On admission demographic details for these patients were also recorded. This included the patient's abbreviated mental test score, which is a series of ten questions of mental ability [20]. Physical state was assessed using the American Society of Anaesthetists (ASA) grade [2]. The ability of the patient to walk about their own home without walking aids was also recorded along with the patient's usual residence which was divided into those living in their own homes and those in institutional care.

In total 1255 patients were treated by fixation of the fracture with three $6.5 \mathrm{~mm}$ cancellous screws. The indications for internal fixation were all undisplaced and partially displaced fractures and displaced fractures in those aged less than 70 years of age. In addition, selected patients over 70 years of age with a displaced intracapsular fracture were also treated by internal fixation. These included patients who were felt to be very active and physiologically fit for their age, the very frail in which arthroplasty was considered a too extensive procedure, those with a potential site of sepsis and those with a bleeding disorder.

Undisplaced fractures were fixed in situ. Partially displaced and displaced fractures were reduced under image intensification in theater with the fracture table. First, the fracture was reduced if required by gentle traction whilst screening in the AP radiograph. Then the fracture was reduced if necessary in the lateral view by internal rotation of the limb. The aim was to achieve a Garden reduction angle of 160 to 170 degrees on the AP film and a reduction angle as close to 180 degrees on the lateral radiograph [9]. Post-operative rehabilitation was standardized with immediate full-weight bearing in those patients over 60, and six to eight weeks partial weight bearing in those aged less than 60 with a displaced fracture.

After discharge from hospital, all patients were then followed up in a hip fracture clinic. Patients were normally reviewed three or four times till one year from injury. For those unable to attend repeated follow-up visits then final follow-up was by telephone interview at one year from injury. Any patients with significant residual symptoms in the hip were invited to re-attend the clinic. Non-union was defined as either re-displacement (or displacement for an initially undisplaced fracture) or failure of evidence of bony union on either the AP or the lateral radiograph by one year post-fixation. Avascular necrosis was defined as any grade II to IV of necrosis during any of the follow-up periods [22]. Patients were excluded from the study if they did not have a minimum of 90 days radiographic follow-up, unless they developed fracture healing complications within this time period in which case they were included.

\begin{tabular}{lccc}
\hline & Undisplaced & $\begin{array}{c}\text { Partially } \\
\text { displaced }\end{array}$ & $\begin{array}{c}\text { Fully } \\
\text { displaced }\end{array}$ \\
\hline Number of patients & 259 & 106 & 513 \\
\hline Mean age (range) & 73.2 & 77.1 & $(22-96)$ \\
$(16-99)$ & $(37-100)$ & $(22-96)$ \\
\hline Number female (\%) & $208(80.3)$ & $88(83.0)$ & $386(75.2)$ \\
\hline Independently mobile (\%) & $192(74.1)$ & $69(65.1)$ & $378(73.7)$ \\
\hline Mean mental test score (/10) & 6.9 & 6.6 & 6.9 \\
\hline From own home (\%) & $220(84.9)$ & $80(75.4)$ & $425(82.8)$ \\
\hline Mean (median) ASA grade & $2.4(2)$ & $2.5(2)$ & $2.5(2)$ \\
\hline
\end{tabular}

Table 1: Characteristics of patients.

Statistical analysis between groups was performed on GraphPad InStat version 3.00 for Window 95, (GraphPad Software, San Diego, California, USA), using the $t$-test for continuous outcomes and the Fisher exact test for binary outcomes. A $P$-value of less than .05 was considered as statistically significant.

\section{Results}

Of the original 1255 patients treated with internal fixation, 377 patients were excluded. The numbers of patients excluded in each group were $224(46.2 \%)$ of the undisplaced group, 42 (28.4\%) of the partially displaced group, and 111 $(17.8 \%)$ of the displaced group. In total 878 patients were therefore included in the study, and were followed for a mean radiographic follow-up of 368 days.

Table 1 lists the characteristics of the patient for the three fracture types. There was a significantly greater proportion of patients from their own home for those with undisplaced fractures in comparison to those with partially displaced fractures $(P=.03)$. In addition, those patients with a partially displaced fracture were significantly older than the other groups ( $P$-value for the differences .002 and .01). There was also tendency for those with a partially displaced fracture to be more likely to have impaired mobility, although the differences between the groups were not statistically significant.

Table 2 displays the treatment methods and characteristics of all the patients with intracapsular hip fractures treated at this center over the study period. This gives some demographic details of all patients treated with hemiarthroplasty. It also lists all those excluded from the study (for reasons given), none of which developed fracture healing complications.

Table 3 details the occurrence of fracture healing complications related to the degree of fracture displacement, with a subdivision into age ranges within each group. The displayed $P$-values were calculated in comparing the different groups against the displaced fracture figures. For the complications on non-union and total fracture 


\begin{tabular}{lcccc}
\hline \multirow{2}{*}{ Treatment used } & Hemiarthroplasty & \multicolumn{3}{c}{ Internal fixation } \\
\cline { 3 - 5 } & & $\begin{array}{l}\text { Excluded as died without } \\
\text { adequate radiological follow-up }\end{array}$ & $\begin{array}{l}\text { Excluded as unable to attend } \\
\text { for radiological follow-up }\end{array}$ & Included in this study \\
\hline Number & 1742 & 125 & 252 & 878 \\
\hline Mean age (range) & $82.3(47-104)$ & $83.1(59-99)$ & $79.8(28-103)$ & $73.5(16-100)$ \\
\hline Number male (\%) & $306(17.6 \%)$ & $43(3.4 \%)$ & $44(17.5 \%)$ & $197(22 \%)$ \\
\hline Mean (median) ASA grade & $2.7(3)$ & $3.4(3)$ & $2.6(3)$ & $2.5(2)$ \\
\hline Living in institution $(\%)$ & $485(27.8 \%)$ & $61(48.8 \%)$ & $88(34.9 \%)$ & $144(16.4 \%)$ \\
\hline
\end{tabular}

Table 2: Treatment methods and characteristics of all patients with intracapsular hip fractures treated over the study period.

\begin{tabular}{|c|c|c|c|c|c|}
\hline & Age range & Number of fractures & Non-union & Avascular necrosis & All fracture healing complications \\
\hline \multirow{4}{*}{ Undisplaced (\%) } & $<60$ & 48 & $0(0)$ & $2(4.2)$ & $2(4.2)$ \\
\hline & $60-70$ & 42 & $3(7.1)$ & $1(2.4)$ & $4(9.5)$ \\
\hline & $>70$ & 169 & $29(17.2)$ & $15(8.9)$ & $44(26.1)$ \\
\hline & All patients & 259 & $32(12.3 \%) P<.0001$ & $18(6.9 \%) P=.34$ & $60(23.2 \%) P<.0001$ \\
\hline \multirow{4}{*}{ Partially displaced (\%) } & $<60$ & 7 & $1(14.3)$ & $1(14.3)$ & $2(28.6)$ \\
\hline & $60-70$ & 14 & $0(0)$ & $1(7.1)$ & $1(7.1)$ \\
\hline & $>70$ & 85 & $26(30.6)$ & $4(4.7)$ & $30(35.3)$ \\
\hline & All patients & 106 & $27(25.7 \%) P=.03$ & $6(5.7 \%) P=.34$ & $33(31.1 \%) P=.007$ \\
\hline \multirow{4}{*}{ Fully displaced (\%) } & $<60$ & 74 & $13(17.6)$ & $14(18.9)$ & $27(36.5)$ \\
\hline & $60-70$ & 134 & $45(33.6)$ & $13(9.7)$ & $57(42.5)$ \\
\hline & $>70$ & 305 & $129(42.3)$ & $20(6.6)$ & 149 (48.9) \\
\hline & All patients & 513 & $187(36.4 \%) P<.0001$ & $47(9.1 \%) P=.21$ & $234(45.6 \%) P<.0001$ \\
\hline
\end{tabular}

Table 3: Incidence of fracture non-union and avascular necrosis related to the age of the patient and displacement of the fracture $(P$-values displayed for comparison of complication rates for undisplaced and partially displaced groups versus fully displaced).

healing complications, the difference between either the undisplaced or partially displaced in comparison to those with a displaced fracture was statistically significant, with an increase in the complication rate as the fracture became more displaced. There was a similar distribution of age ranges for each of the groups. There was a slightly higher proportion of avascular necrosis complications and lower non-union rates in the younger patients, however the numbers were too small for comparison.

The graph in Figure 4 displays the relationship between the trabecular angles as measured on the lateral radiograph on admission. For a trabecular angle below 150 degrees the rate of complications was high at $46 \%$. For 150 to 170 degrees this fell to $38 \%$, and for 170 degrees and above the complication rate was the lowest at $25 \%$.

\section{Discussion}

The aim of our study was to classify and evaluate internal fixation of intra-capsular neck of femur fractures with consideration to the degree of displacement on both the AP and lateral radiographs. Displacement was defined by separation of the cortex at the fracture line. In order to give a range of displacements, and therefore a comparison between those

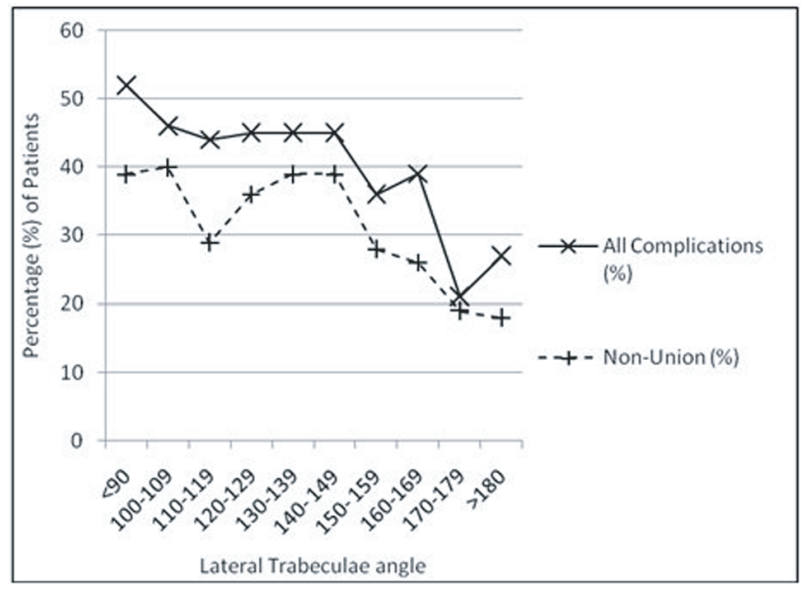

Figure 4: Graph showing complications related to lateral radiograph trabecular angle (normal angle 180 degrees).

ranges, we revisited Linton's previously described progression of fracture displacement, dividing intra-capsular fractures into three subgroups: undisplaced, partially displaced and displaced. The group of undisplaced fractures were selfdefining with no fracture displacement on either radiograph. 
The partially displaced fracture is based on both AP and lateral radiographs as the fracture is therefore displaced on one, but not both, of the radiograph modalities. Generally, these partially displaced fractures were those that showed angulation on the lateral radiograph, but in some cases the displacement was on the AP radiograph. The displaced fractures displayed cortical displacement on both radiograph modalities. The partially displaced fractures have complication rates inbetween that of undisplaced fractures and displaced fractures.

There have been many previous descriptions and classifications for femoral neck fractures. The majority of these have been based on the radiographic appearance of the fracture on the anteroposterior (AP) radiograph, which to some extent ignores the information given by the lateral radiograph. Garden, in 1964, described intracapsular fractures in terms of the angulation of the trabeculae solely on the AP radiograph [9]. He described four stages of fracture displacement, un-displaced incomplete (stage 1), un-displaced complete (stage 2), complete fracture, incompletely displaced (stage 3) and complete fracture completely displaced (stage 4). This grading method has since been found to be unreliable in predicting fracture healing complications for the four groups. This classification method is now only used to divide fractures into two broad categories: displaced and undisplaced groups $[8,16]$. Pauwels' classification used the angle of the fracture line on the AP radiograph to produce three groups [19]. Undisplaced fractures were invariably grade 1 and displaced fractures grades II and III. Again this classification method has been shown to be of little practice use [17]. The more recent AO classification divides intracapsular fractures into nine groups based on the AP appearance of the fracture and the angle of the fracture line $[14,15]$. Evaluations of the value of this classification method have consistently reported it to have an unacceptable degree of inter- and intra-observer variation $[5,15]$.

Many previous reports on intracapsular fractures have used the term impacted fracture for a Garden grade 1 fracture $[4,6,10,21]$. Such a fracture from a post-mortem is shown in Figure 5. As can be seen, there is in fact no impaction of the fracture, just displacement of the fracture on the lateral view which would lead to the radiographic appearance that could be misinterpreted as impaction on the AP radiograph, and therefore classically defined as an undisplaced fracture. Impaction of the fracture, implying some stability to resist displacement, may therefore be an incorrect term, as the fracture represents the first stage of a possible progressive displacement for this fracture if not stabilized, or may under-estimate the possibility of complication following stabilization.

The overall rate of fracture healing complications in this study may be higher than in some previous reports in

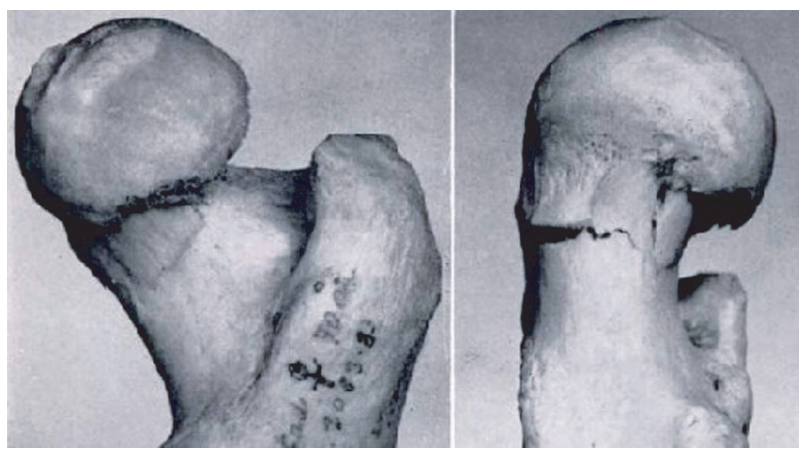

Figure 5: Post-mortem specimen of an "impacted" intracapsular fracture demonstrating how the displacement of the fracture on the lateral view leads to an apparent impaction on the fracture on the anterior-posterior view. (photograph from [12]).

the literature. This is because it is not a consecutive series of patients. Within this series, we excluded 377 patients who either died or failed to attend the follow-up in order to be able to achieve adequate radiographic follow-up. This produced a high exclusion rate of around $30 \%$ overall of patients in which no complications were known to have occurred. We did however include any patient who was known to have developed fracture complications at any time after surgery including those within the 90 day follow-up cut-off. In our study, we therefore have a very positive selection towards inclusion of patients with complications. Therefore, the overall reported risk of complication will be marginally higher than that which occurs for a consecutive series of patients which includes patients who have died or been lost to follow-up shortly after surgery as has been applied to previous studies. A summary of previously published studies has reported a non-union rate of $33 \%$ and an incidence of avascular necrosis of $16 \%$ for displaced fractures and a non-union rate of $4 \%$ and avascular necrosis rate of $2 \%$ for undisplaced fractures $[7,13]$.

Problems of the methodology of this study are related to the inadequate follow-up for some patients, and not being able to include all patients. Much of this problem related to the very elderly being unable to attend for radiographic follow-up. To restrict the study to only those patients with a longer follow-up would considerably reduce the patients' numbers and make the finding only relevant to a subgroup of younger and fitter patients, able to attend follow-up. Although 90 days may be considered as too short for a radiographic follow-up, any patient with significant symptoms at the time of the telephone follow-up was offered to attend for out-patient review with radiographs. In addition, we were the only hospital within the region treating patients with proximal femoral fractures, so if complications did occur necessitating re-admission it was 
invariable that this was at our hospital. For this group of patients who break the hip, only a small number of patients move out of our hospital catchment area. The numbers excluded for the 3 different groups show a higher number excluded from the undisplaced group. This may be due to the probable absence of symptoms at telephone follow-up and subsequent early discharge which would not allow them to meet the inclusion criteria for the study. This is may therefore contribute to the higher rate of complications particularly in the undisplaced group when compared to previous studies, as the rate of complication in those excluded is likely to be low for the reasons stated above.

The three groups of patients with the different fractures were not identical. Those patients with a partially displaced fracture had a tendency to be older and to be less mobile and more likely to come from institutional care. There is a tendency for the incidence of a fracture non-union to increase with age $[3,18]$. However, the mean difference of only four years increase for the partially displaced fractures is unlikely to have a substantial effect on the occurrence of non-union.

There is continuing controversy regarding the optimum method of treatment for an intracapsular neck of femur fracture, with continuing debate between arthroplasty and internal fixation. The use of hemi-arthroplasty and total hip arthroplasty has many advocates, but there are many advantages to the use of internal fixation including shorter, less invasive operations with less blood loss and lower infection rates. The disadvantages include non-union, and avascular necrosis. It is possible that reduction of the undisplaced or partially displaced fractures may contribute towards non-union and avascular necrosis rates due to added insult to the femoral head blood supply. With knowledge of the complication rates for partially displaced fractures, the surgeon can be more informed for his decision on fracture management. At present, we feel that the complication rate for both undisplaced and partially displaced fractures is sufficiently low to continue to justify internal fixation at the treatment of choice.

In conclusion, femoral neck fractures can show a range of displacement at presentation from undisplaced to fully displaced, with a progressive increase in incidence of fracture healing complications with increasing displacement. Consideration of fracture displacement should be based on both anteroposterior and lateral radiographs.

\section{References}

[1] A. Alho, J. G. Benterud, H. Ronningen, and A. Hoiseth, Prediction of disturbed healing in femoral neck fracture: radiographic analysis of 149 cases, Acta Orthop Scand, 63 (1992), 639-644.

[2] American Society of Anesthesiologists, New classification of physical status, Anesthesiology, 24 (1963), 111.

[3] R. Barnes, J. T. Brown, R. S. Garden, and E. A. Nicoll, Subcapital fractures of the femur: a prospective review, J Bone Joint Surg Br, 58-B (1976), 2-24.
[4] G. Bentley, Impacted fractures of the neck of the femur, J Bone Joint Surg Br, 50-B (1968), 551-561.

[5] C. M. Blundell, M. J. Parker, G. A. Pryor, J. Hopkinson-Wooley, and S. S. Bonsall, An assessment of the AO classification of intracapsular fractures of the proximal femur, J Bone Joint Surg Br, 80-B (1998), 679-683.

[6] R. E. Bunata, J. J. Fahey, and D. B. Drennan, Factors influencing stability and necrosis of impacted femoral neck fractures, JAMA, 223 (1973), 41-44.

[7] K. S. Conn and M. J. Parker, Un-displaced intra-capsular hip fracture: results of internal fixation in 375 patients, Clin Orthop Relat Res, 421 (2004), 249-254.

[8] P. Eliasson, L. I. Hansson, and J. Kärrholm, Displacement in femoral neck fractures: a numerical analysis of 200 fractures, Acta Orthop Scand, 59 (1988), 361-364.

[9] R. S. Garden, Stability and union in subcapital fractures of the femur, J Bone and Joint Surgery Br, 46-B (1964), 630-647.

[10] B. A. Hansen and S. Solgaard, Impacted fractures of the femoral neck treated by early mobilization and weight-bearing, Acta Orthop Scand, 49 (1978), 180-185.

[11] P. Linton, On the different types of intracapsular fractures of femoral neck: a surgical investigation of origin, treatment, prognosis and complications in 365 cases, Acta Chir Scand, 90 (1944), 1-122.

[12] P. Linton, Types of displacement in fractures of the femoral neck and observations on impaction of fractures, J Bone Joint Surg Br, 31-B (1949), 184-189.

[13] G. L. Lu-Yao, R. B. Keller, B. Littenberg, and J. E. Wennberg, Outcomes after displaced fractures of the femoral neck: a metaanalysis of one hundred and six published reports, J Bone Joint Surg Am, 76-A (1994), 15-25.

[14] M. E. Müller, S. Nazarian, P. Koch, and J. Schatzker, The Comprehensive Classification of Fracture of Long Bones, SpringerVerlag, Berlin, 1st ed., 1990.

[15] M. L. Newey, D. Ricketts, and L. Roberts, The AO classification of long bone fractures; an early study of its use in clinical practice, Injury, 24 (1993), 309-312.

[16] M. J. Parker, Garden grading of intracapsular fractures: meaningful or misleading?, Injury, 24 (1993), 241-242.

[17] M. J. Parker and E. Dynam, Is Pauwels classification still valid?, Injury, 29 (1998), 521-523.

[18] M. J. Parker, R. Raghavan, and K. Gurusamy, Incidence of fracture-healing complications after femoral neck fractures, Clin Orthop Relat Res, 458 (2007), 175-179.

[19] F. Pauwels, Der Schenkelhalsbruch, ein Mechanisches Problem: Grundlagen des Heilungsvorganges, Prognose und Kausale Therapie, vol. 63 of Zeitschrift für Orthopädische Chirurgie, Einschliesslich der Heilgymnastik und Massage, Ferdinand Enke Verlag, Stuttgart, Germany, 1935.

[20] K. N. Qureshi and H. M. Hodkinson, Evaluation of a ten-question mental test in the institutionalized elderly, Age Ageing, 3 (1974), $152-157$.

[21] E. L. F. B. Raaymakers and R. K. Marti, Non-operative treatment of impacted femoral neck fractures: a prospective study of 170 cases, J Bone Joint Surg Br, 73-B (1991), 950-954.

[22] M. E. Steinberg, G. D. Hayken, and D. R. Steinberg, A quantitative system for staging avascular necrosis, J Bone Joint Surg Br, 77-B (1995), 34-41. 